\title{
Proyectos de ingeniería integrados a la comunidad (PIIC), una visión institucional y metodológica
}

\author{
Jaime Andrés Vélez, Franklin Jaramillo, Julio César Saldarriaga \& Carlos Alberto Palacio \\ Facultad de Ingeniería, Universidad de Antioquia, Medellín, Colombia. jaime.velezz@udea.edu.co, franklin.jaramillo@udea.edu.co, \\ julio.saldarriaga@udea.edu.co,cpalacio@udea.edu.co
}

\begin{abstract}
Resumen - Se presenta la estructura metodológica del curso "Proyectos de Ingeniería Integrados a la Comunidad" (PIIC), como parte de una estrategia de la Facultad de Ingeniería de la Universidad de Antioquia para efectuar las directrices de su plan de desarrollo en cuanto a la implementación de procesos innovadores en la enseñanza de la Ingeniería. Con esta metodología se responde también a la necesidad de acercar a los estudiantes a la comunidad y ofrecerle a ambos frentes (estudiantes y comunidad) la oportunidad de participar en proyectos de ingeniería en todas sus fases, a través de la aplicación de técnicas pedagógicas basadas en la resolución de problemas, trabajo multidisciplinario, conformación de grupos multinacionales y multiculturales y el uso de las TIC. Se desglosan los distintos proyectos realizados hasta la fecha y se destaca la importancia de la creación de redes y el apoyo institucional en el éxito de este tipo de iniciativas.
\end{abstract}

Palabras Clave- Impacto social, pedagogía, vocación ingenieril, proyectos ingeniería.

Recibido:14 de octubre de 2016. Revisado: 28 de noviembre de 2016. Aceptado: 09 de diciembre de 2016.

\section{Engineering proyects integrated to the community (PIIC)institutional and methodological visión}

\begin{abstract}
The methodology of the course "Engineering Projects Integrated to the Community" (PIIC) is presented as a response of the Faculty of Engineering of University of Antioquia to the need of closing the gap between engineering students and the community while, at the same time, offering to both students and the community the opportunity of participating in an engineering project in all its phases. This will be accomplished through the application of pedagogic techniques based in the problem solution, multidisciplinary work, and conformation of multinational and multicultural groups and the use of ICT (Information and Communication Technologies). The different projects developed to date are detailed and the importance of the network building and the institutional support in this type of initiatives is highlighted.
\end{abstract}

Keywords - Social impact, pedagogy, engineering calling, engineering projects.

\section{Introducción}

El papel de la ingeniería en el desarrollo de las sociedades y en el innegable avance en la calidad de vida a nivel mundial ha sido crucial. Sin embargo, este desarrollo no es uniforme y genera sus propios problemas en cuanto a inequidad, reducción de la pobreza, cambio climático y desarrollo sostenible, entre otros muchos.

Es fundamental entonces, asumir estos retos sociales y ambientales como parte integral del quehacer ingenieril y no como un objetivo secundario o un subproducto más o menos deseable del desarrollo económico. El profundo efecto de las nuevas técnicas y tecnologías aplicadas en las vidas de las personas corrientes no puede dejarse al azar, debe ser tema de constante reflexión y reevaluación, no solo en el ámbito del ejercicio de la profesión ingenieril, sino desde la fuente misma, es decir, las aulas donde se forman las nuevas generaciones de ingenieros y que en la mayoría de los casos, son también el último lugar donde se tiene la oportunidad de influir sobre el tipo de ingenieros que nuestras sociedades requieren.

Este debate no es nuevo, la ingeniería ha sido definida por la Accreditation Board for Engineering and Technology (ABET) como: "La profesión en la cual el conocimiento de las matemáticas y las ciencias naturales ganado a través del estudio, la experiencia y la práctica, es aplicado conscientemente en el desarrollo de formas económicas de uso de los materiales y las fuerzas de la naturaleza para el beneficio de la humanidad'[1] (énfasis añadido por los autores).

Sin embargo, existen factores que hacen que este llamado al bien común no esté tan insertado en la práctica del oficio como sería deseable y entre ellos están:

\subsection{El bajo conocimiento social de la labor del ingeniero}

A pesar del papel crucial que desempeña el ingeniero en el desarrollo económico, parece existir una desconexión entre su trabajo y el producto final tal como es percibido por la sociedad [2]. Esto se debe a la posición tradicional del ingeniero dentro de la cadena productiva (Figura 1), donde rara vez se relaciona de forma directa con la sociedad, sea como el que atiende sus necesidades, o como quien finalmente entrega los productos requeridos.

La desconexión a la que se hace alusión, funciona en ambos sentidos, primero la sociedad no identifica con claridad el mérito del trabajo del ingeniero, al quedar éste sepultado en medio del sistema productivo y segundo, a su vez el ingeniero no comprende en muchas ocasiones el impacto último de su labor en la sociedad a la que sirve.

Como citar este artículo: Vélez, J. A., Jaramillo, F., Saldarriaga, J. C. \& Palacio, C. A.. Proyectos de ingeniería integrados a la comunidad (PIIC), una visión institucional y metodológica. Revista Educación en Ingeniería 12 (23) 76-82, Febrero, 2017. 


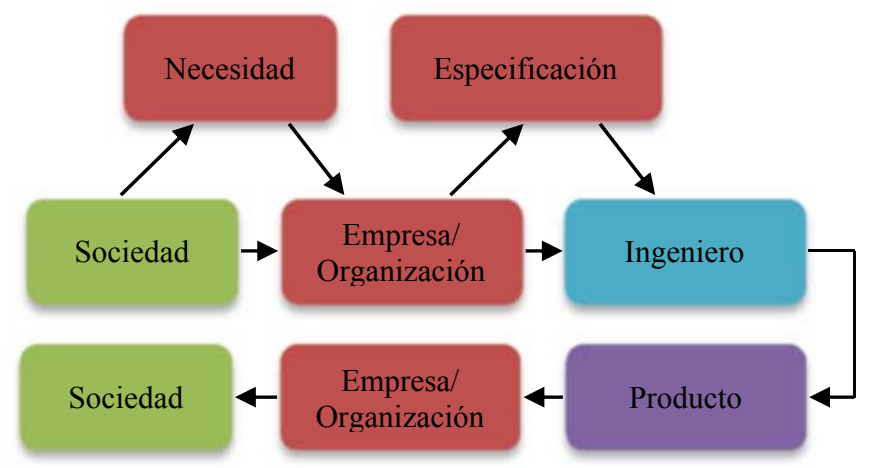

Figura 1. Representación esquemática de la posición del ingeniero en la cadena productiva.

Fuente: Los autores.

\subsection{Segregación cultural}

El ejercicio de la ingeniería implica el dominio de conocimientos altamente especializados, adquiridos durante años de formación intensiva que necesariamente separan al ingeniero culturalmente del promedio de la población, lo que invita por un lado al desarrollo de actitudes elitistas y por el otro a la desconfianza de la población que ve al ingeniero como un ente misterioso y aislado o apático. Esta separación puede comenzar desde la academia en casos extremos, cuando los procesos de enseñanza tradicionales priorizan en exceso los principios técnicos en detrimento de los valores sociales [3]

\subsection{Reconocimiento Social}

Producto de lo anterior, la extremada especialización requerida por los diferentes campos de la ingeniería, no solo distancia al ingeniero de la sociedad, sino que separa a los propios ingenieros entre sí, de acuerdo con su área de experticia, lo que dificulta la coalescencia del grupo en causas comunes, así como la eficacia a la hora de resolver problemas complejos que requieran conocimientos en diversas disciplinas.

Las anteriores son apenas algunas de las barreras a salvar si se quiere alcanzar el ideal de un ingeniero plenamente consciente de su capacidad como agente del cambio social positivo y, aunque hay muchas asociaciones y organizaciones dedicadas a propiciar este cambio de paradigma, la magnitud de la tarea hace que difícilmente sean suficientes, sobre todo si no se ataca el problema desde las mismas aulas [4].

Como un intento de mitigar algunos de estos factores a nivel local en el municipio de Medellín y en el departamento de Antioquia, surgió la iniciativa de Proyectos de Ingeniería Integrados a la Comunidad (PIIC) de la Facultad de Ingeniería de la Universidad de Antioquia, experiencia que será presentada a lo largo de este artículo.

\section{Antecedentes}

La idea seminal del curso PIIC se basa en el modelo Engineering Projects In Community Service [5] (EPICS $\left.{ }^{\circledR}\right)$ de la Universidad de Purdue, donde la idea central consiste en el desarrollo de proyectos multidisciplinarios en los cuales los alumnos deben responder a las necesidades de un cliente que es, en este caso, la comunidad. Este modelo fue expuesto por el director del programa EPICS William Oakes durante la celebración de un taller en Medellín en el año 2012, al cual fueron invitadas diversas instituciones de la ciudad.

A partir de ese taller, los autores, con el aval de las directivas de la Facultad de Ingeniería, establecieron un estrecho contacto con el grupo EPICS ${ }^{\circledR}$ de la Universidad de Purdue, que desembocó primero en la creación de un grupo de trabajo PIIC conformado por alumnos de ingeniería mecánica y de materiales en 2013, seguido en 2014 por la propuesta formal del curso al Consejo de la Facultad de Ingeniería donde fue aprobado unánimemente como curso electivo de Facultad. Finalmente se logró ese mismo año el reconocimiento por parte de la Universidad de Purdue al curso PIIC como EPICS Global Partner.

Como respuesta a este nuevo proyecto de inclusión de procesos enfocados a la formación de ingenieros, tras visitas a universidades del exterior y a partir de las experiencias exitosas de PIIC, en el año 2015 se crea la Unidad de Pedagogía en Ingeniería (UPI), que representa un gran paso adelante en la integración de metodologías innovadoras a la enseñanza de la ingeniería en la Universidad de Antioquia, siendo PIIC el laboratorio ideal para la prueba de estas metodologías con miras a su adopción posterior en otros cursos de la Facultad. Esta Unidad plantea apuestas tanto en la formación de estudiantes, como la necesidad de evaluar los procesos actuales de enseñanza, aspectos en los cuales se concentra la iniciativa PIIC.

\section{Metodología}

Si bien los principios básicos de multidisciplinariedad y servicio comunitario de EPICS son compartidos por el curso PIIC, las diferencias en cuanto al número de estudiantes, la duración del programa y los recursos disponibles, hacen que las metodologías por necesidad sean diferentes, por lo que hoy en día el curso PIIC cuenta con una identidad y metodología propios, diseñados para aprovechar de forma eficiente los recursos particulares de la institución en la que opera y las comunidades a las que sirve.

Los objetivos principales de PIIC son:

- Acercar los estudiantes a la comunidad, fomentando la interacción personal con individuos de todos los estratos sociales.

- Visibilizar a los estudiantes de ingeniería en las comunidades, permitiendo que estas conozcan de primera mano el quehacer ingenieril.

- Presentar el estudio de la ingeniería como un camino cercano y viable de desarrollo personal para los habitantes de la comunidad.

- Ofrecer tanto a los estudiantes como a los habitantes de la comunidad, la oportunidad de participar en el ciclo completo de un proyecto de ingeniería. Acá se conciben desde la fase de exploración del problema, hasta la fase de implementación de las soluciones, eliminando barreras culturales y fomentando el sentido de pertenencia tanto de 
alumnos como de la comunidad, al verse a sí mismos como parte del mismo tejido social.

- Sacar a los estudiantes de su zona de confort (el aula), enfrentándolos a entornos de trabajo colaborativo interdisciplinario, multinacional y multicultural en los que deberán desarrollar habilidades adicionales esenciales para el futuro ejercicio de su profesión como, por ejemplo, la habilidad para la transmisión efectiva de sus ideas, el manejo de lenguas extranjeras o la capacidad de generar empatía con otras culturas, entre otras.

El resultado final de la metodología PIIC es exponer al estudiante a un cambio en el paradigma tradicional del esquema productivo, lo que se resume en la Figura 1, donde se reemplaza por la interacción inmediata y transparente de todos los actores: ingeniero, producto (entendiéndose éste como una respuesta concreta a una necesidad) y la sociedad (Fig. 2).

Bajo este nuevo paradigma, el estudiante se ve involucrado directamente con el resultado de su labor y puede comprobar de primera mano el impacto que su trabajo tiene en la comunidad, lo que estimula el surgimiento de un sentido de responsabilidad social y ayuda en su formación integral, tras reforzar las conocidas habilidades blandas.

Para alcanzar estos objetivos, es necesario implementar un modelo pedagógico e institucional capaz de suministrar a alumnos y docentes, la estructura, la flexibilidad y los recursos necesarios sin sacrificar rigor en el proceso formativo. A continuación, se detallan las características principales de estos dos modelos, así como la comunidad intervenida y los casos de aplicación.

\subsection{Modelo pedagógico}

Según el Documento Rector de Transformación Curricular de la Facultad de Ingeniería de la Universidad de Antioquia (2006), implementado en los programas académicos hacia el año 2008, la transversalidad del Currículo implica los siguientes conceptos y procedimientos [6]:

- Formación en investigación: Todos los proyectos de aula se constituyen bajo la formulación de problemas que posibilitan la estructura metodológica para su solución, potenciando las competencias de los estudiantes en el campo de la investigación.

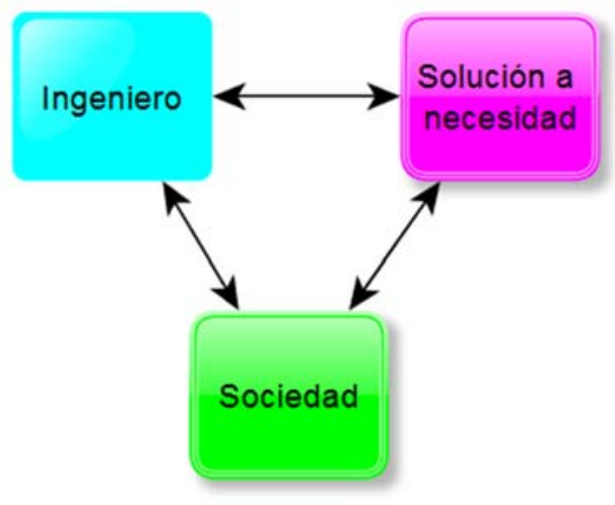

Figura 2. Esquema productivo PIIC.

Fuente: Los autores.
- Competencias Comunicativas: Todos los proyectos de aula incorporan, en alguna medida, las competencias de leer, escribir, escuchar y exponer.

- La Formación Integral: Todos los proyectos de aula propenden por el desarrollo de la inteligencia, que se vislumbra en la potencialización de competencias por la adquisición de conocimientos y por la incorporación de valores y sentimientos, que se explicitan a través de los conocimientos en el mejoramiento cualitativo del ser social.

- Uso de nuevas Tecnologías: Todos los proyectos de aula en alguna medida, harán uso de las nuevas tecnologías.

- Normas de Aseguramiento de la Calidad: Algunos proyectos de aula en sus conceptos y procedimientos integrarán la aplicación pertinente de las normas, regulaciones y estandarización, definidas a nivel nacional e internacional.

- Emprendimiento y Empresarismo: En todos los proyectos de aula debe estimularse la creación de nuevas ideas, orientadas al desarrollo de la investigación para la creación de empresas y no constreñir este ideal al desarrollo de cursos aislados sobre el tema.

- Lenguas Extranjeras: Los proyectos de aula basarán sus fuentes bibliográficas en Lenguas Extranjeras, en la medida en que el desarrollo de los conocimientos así lo exijan.

Con el fin de promover la integración al aula de estos principios, se recurre a la formulación de una metodología de Aprendizaje Basado en Problemas y Proyectos (Problem and Project Based Learning, PLB. Ver [7]) adaptada a las particularidades del curso PIIC y que tiene las siguientes características:

- El problema es el punto de partida del proceso formativo y, en el caso de PIIC, surge directamente de la interacción de los estudiantes con la comunidad bajo principios tomados de la Investigación Acción Participativa (IAP) [8] y la Investigación e Innovación Responsable (RRI) [9].

- La aproximación a la comunidad se hace previa capacitación por parte de asesores expertos en Trabajo Social, de tal forma que la interacción se establezca desde la igualdad y la empatía, así como para evitar malos entendidos que puedan dificultar la ejecución del proyecto. El estudiante debe verse como parte de la comunidad a la que sirve.

- Los estudiantes aprenden por experiencia directa, al formular hipótesis y ponerlas a prueba durante el desarrollo del proyecto, lo que implica que los estudiantes deben realizar actividades de investigación, planeación, escritura, prototipos, y presentaciones que estimulan el aprendizaje en profundidad y de manera integral.

- Los proyectos son llevados a cabo por grupos de estudiantes (típicamente de entre 3-5 alumnos) provenientes de todas las carreras ofrecidas en la Facultad de Ingeniería. Se fomenta en ellos, la interdisciplinariedad y la cooperación en la formulación de aproximaciones a la solución del problema objeto de estudio, desde múltiples áreas del conocimiento ingenieril.

- Cada proyecto cuenta, además de la guía del docente PIIC, con un banco de asesores externos expertos en campos particulares a los cuales los alumnos pueden acudir y que, además, participan en los procesos de evaluación. 
- Los grupos de trabajo PIIC en la universidad de Antioquia, cuentan a su vez con grupos espejo en otras instituciones nacionales o internacionales, los cuales hacen parte integral del desarrollo del proyecto y de la formación del ingeniero.

- De ser necesario, se crean alianzas estratégicas con grupos de investigación y laboratorios dentro y fuera de la Facultad, con el fin de realizar los experimentos o pruebas que el proyecto requiera.

- De acuerdo con Cross (2008) y Pahl, Wallace, \& Bessing (2007), el marco teórico que integra a las diferentes disciplinas en un flujo de trabajo coherente desde la definición del problema hasta la implementación de la solución, es el del diseño ingenieril.

- La duración del curso lectivo es de un semestre académico (16 semanas), sin embargo, si el proyecto lo amerita, puede continuarse al semestre siguiente con un grupo nuevo de estudiantes, asesorados por voluntarios del semestre inmediatamente anterior.

- Seguimiento efectivo: Un grupo de trabajo eficiente debe tener bien definidas $\mathrm{y}$ en cada momento, las responsabilidades de cada uno de sus miembros y, además, contar con las herramientas que hagan esta división de tareas transparente, de tal forma que cada uno de los integrantes, así como el docente, sepa qué están haciendo los demás en todo momento. Es decir, cuales son las fechas de vencimiento de cada actividad y como sus propias actividades se enmarcan dentro del proceso global del proyecto. El curso PIIC integra este principio en su metodología, aplicando una variación del sistema de Flujo ágil de desarrollo de software que se implementa como metodología Kanban [12] en la plataforma web de trabajo colaborativo Trello.Inc [13] (Ver Fig. 3).

La estructura general de una sesión de trabajo consta de un segmento de seguimiento y socialización de las tareas llevadas a cabo, un segmento de trabajo grupal donde los alumnos acompañados del asesor evalúan y reparten actividades y un segmento de clase magistral en metodología del diseño. Los tiempos asignados a cada segmento varían de acuerdo con las necesidades de cada proyecto.

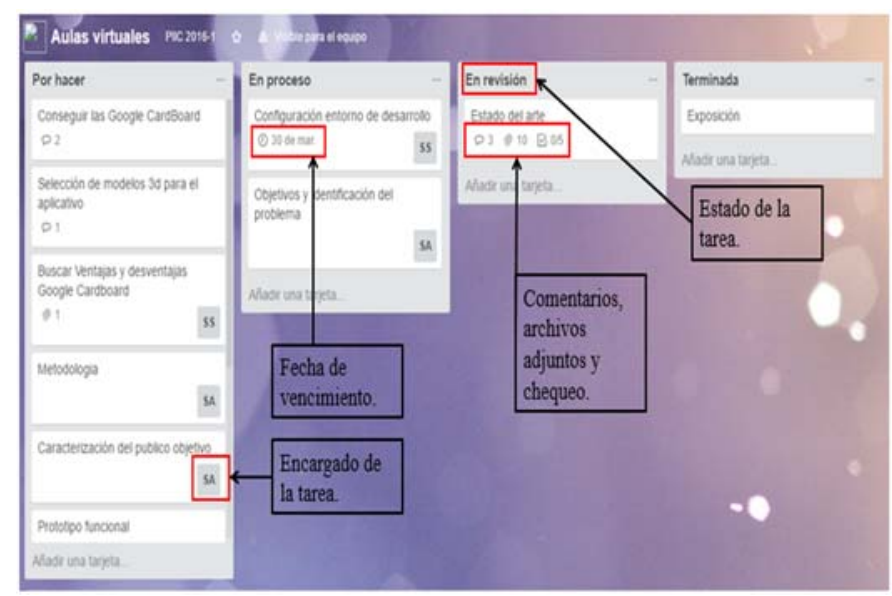

Figura 3. Ejemplo de uso de la metodología Kanban en la plataforma Trello. Fuente: Los autores.

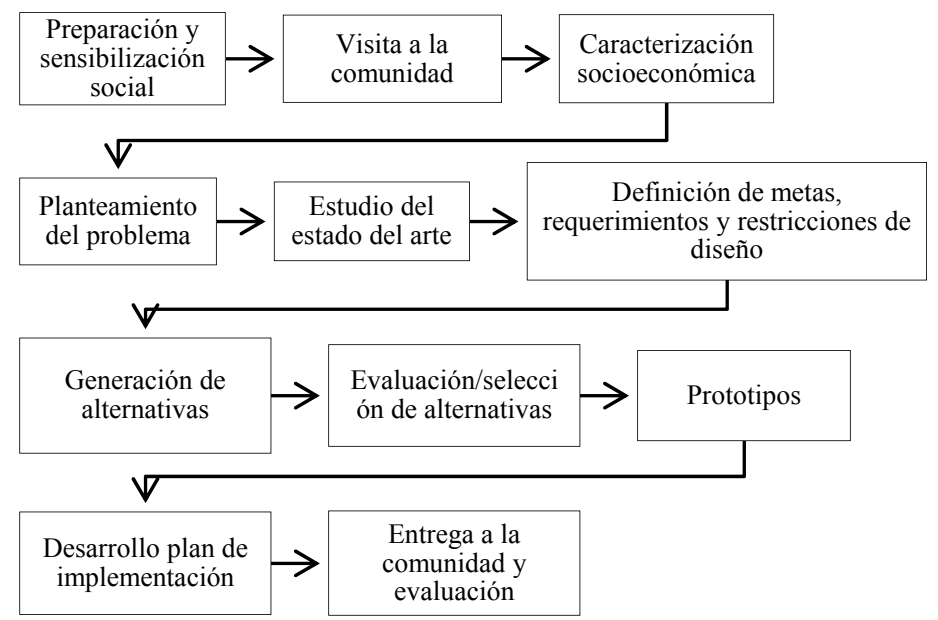

Figura 4. Flujo típico curso PIIC.

Fuente: Los autores.

La evaluación del curso se hace a través de informes escritos y presentaciones orales periódicas de avance grupal y reportes individuales; culminando con un reporte y presentación de cierre del proyecto, ante el docente del curso y los asesores externos.

El estudiante que termina el curso, puede de forma voluntaria continuar en calidad de "egresado" o voluntario, aportando los conocimientos adquiridos durante el desarrollo del proyecto y actuando como asesor y par evaluador en proyectos relacionados.

Las soluciones entregadas a la comunidad deben tener como componente principal la transmisión de conocimiento. Según el proyecto, se pueden instalar prototipos, pero es la propia comunidad quien aprende a implementar y a mantener la solución propuesta.

En líneas generales el proceso de un curso PIIC puede verse resumido según lo mostrado en la Fig. 4.

\subsection{Comunidad intervenida}

El grupo EPICS de la Universidad de Purdue trabajó en los años 2010-2012 con comunidades remotas del Chocó, concretamente en Beté y con los indígenas de Wounaam, sin embargo, las dificultades de acceso y de logística, los llevó a reevaluar la necesidad de contar con un socio estratégico en Colombia con quien pudiese llevar a cabo proyectos de impacto social.

Con base en lo anterior y tras el establecimiento del curso PIIC, se decidió continuar con el desarrollo de soluciones energéticas en instituciones educativas como un proyecto conjunto UDEA-Purdue y se dispuso buscar una institución educativa en la cual llevar a cabo las pruebas del sistema que una vez perfeccionado se instalaría en escuelas remotas.

En este sentido, los alumnos del curso PIIC evaluaron distintas zonas e Instituciones educativas, seleccionando finalmente a Altos de Oriente II. Este sitio de intervención, se caracteriza como una zona de invasión, situada entre los municipios de Bello y Medellín, foco de futuros desarrollos. En entrevistas y observaciones directas de los estudiantes en la comunidad, se halló que esta presenta, además, muchos de los 
problemas típicos de cualquier zona de población vulnerable en Colombia [14], entre ellos:

- Alto porcentaje de población desplazada.

- Carencia de servicios públicos como acueducto o alcantarillado.

- Lejanía del casco urbano y difícil acceso.

- Problemas de legalización de predios.

- Presencia de grupos armados.

- La mayoría de la población se dedica a la economía de subsistencia.

Dentro de dicha zona de estudio, se seleccionó la Institución Educativa León XIII como centro para futuros desarrollos y establecimiento de los ensayos pilotos.

Tabla 1

Proyectos realizados por PIIC hasta la fecha.

\begin{tabular}{|c|c|c|c|c|}
\hline Curso & $\begin{array}{l}\text { Número de } \\
\text { estudiantes }\end{array}$ & Proyecto & $\begin{array}{l}\text { Nivel de } \\
\text { ejecución }\end{array}$ & Alianzas \\
\hline 2013-2 & 4 & $\begin{array}{l}\text { Minicable aéreo } \\
\text { de carga Santo } \\
\text { Domingo }\end{array}$ & $\begin{array}{l}\text { Diseño de } \\
\text { detalle }\end{array}$ & $\begin{array}{l}\text { Ruta N } \\
\text { Proy. } \\
\text { Interchange } \\
\text { Grupo de } \\
\text { Diseño } \\
\text { Mecánico } \\
\text { UDEA }\end{array}$ \\
\hline 2013-2 & 4 & $\begin{array}{l}\text { Energía solar } \\
\text { para I.Es }\end{array}$ & $\begin{array}{l}\text { Diseño de } \\
\text { detalle }\end{array}$ & EPICS \\
\hline 2014-1 & 3 & $\begin{array}{l}\text { Camilla de } \\
\text { primeros } \\
\text { auxilios }\end{array}$ & Entregada & $\begin{array}{l}\text { DAGRED } \\
\text { CIDEMAT } \\
\text { EPICS }\end{array}$ \\
\hline 2014-2 & 4 & $\begin{array}{l}\text { Mobiliario } \\
\text { urbano } \\
\text { energía solar }\end{array}$ & $\begin{array}{l}\text { Diseño de } \\
\text { detalle, } \\
\text { próximo a } \\
\text { implementar }\end{array}$ & $\begin{array}{l}\text { EPICS } \\
\text { CIDEMAT } \\
\text { I.E. León XIII }\end{array}$ \\
\hline 2015-1 & 4 & $\begin{array}{l}\text { Filtros de arena } \\
\text { lenta }\end{array}$ & $\begin{array}{l}\text { Prototipo, } \\
\text { próximo a } \\
\text { implementar }\end{array}$ & $\begin{array}{l}\text { Global } \\
\text { Engineering }\end{array}$ \\
\hline \multirow[t]{4}{*}{ 2015-2 } & 5 & $\begin{array}{ll}\text { Innovación en } & \text { en } \\
\text { sistemas de } & \text { potabilización }\end{array}$ & Prototipos & $\begin{array}{l}\text { Angelo State } \\
\text { University } \\
\text { Global } \\
\text { Engineering }\end{array}$ \\
\hline & 5 & $\begin{array}{l}\text { Transferencia } \\
\text { tecnológica } \\
\text { filtro de arena } \\
\text { lenta }\end{array}$ & $\begin{array}{l}\text { Prototipo } \\
\text { instalado }\end{array}$ & $\begin{array}{l}\text { Global } \\
\text { Engineering }\end{array}$ \\
\hline & 4 & $\begin{array}{l}\text { Escalamiento } \\
\text { de sistema de } \\
\text { filtrado }\end{array}$ & $\begin{array}{l}\text { Diseño de } \\
\text { detalle }\end{array}$ & $\begin{array}{l}\text { Global } \\
\text { Engineering }\end{array}$ \\
\hline & 5 & $\begin{array}{l}\text { Estructuras de } \\
\text { soporte solar, } \\
\text { aulas } \\
\text { biorreguladas }\end{array}$ & $\begin{array}{l}\text { Diseño } \\
\text { conceptual }\end{array}$ & EPICS \\
\hline \multirow[t]{4}{*}{ 2016-1 } & 2 & Aulas virtuales & En curso & Por definir \\
\hline & 3 & $\begin{array}{l}\text { Promoción de la } \\
\text { vocación } \\
\text { ingenieril }\end{array}$ & En curso & $\begin{array}{l}\text { Angelo State } \\
\text { University } \\
\text { Grupo } \\
\text { Ingeniería y } \\
\text { Sociedad } \\
\text { Grupo } \\
\text { LUDENS }\end{array}$ \\
\hline & 4 & $\begin{array}{l}\text { Implementación } \\
\text { filtros de arena } \\
\text { lenta }\end{array}$ & En curso & $\begin{array}{l}\text { Global } \\
\text { Engineering } \\
\text { Grupo GIRAB } \\
\text { Laboratorio de } \\
\text { Mineralurgia } \\
\text { UDEA }\end{array}$ \\
\hline & 4 & $\begin{array}{l}\text { Aulas } \\
\text { Biorreguladas }\end{array}$ & En curso & EPICS \\
\hline
\end{tabular}

Fuente: Los autores.

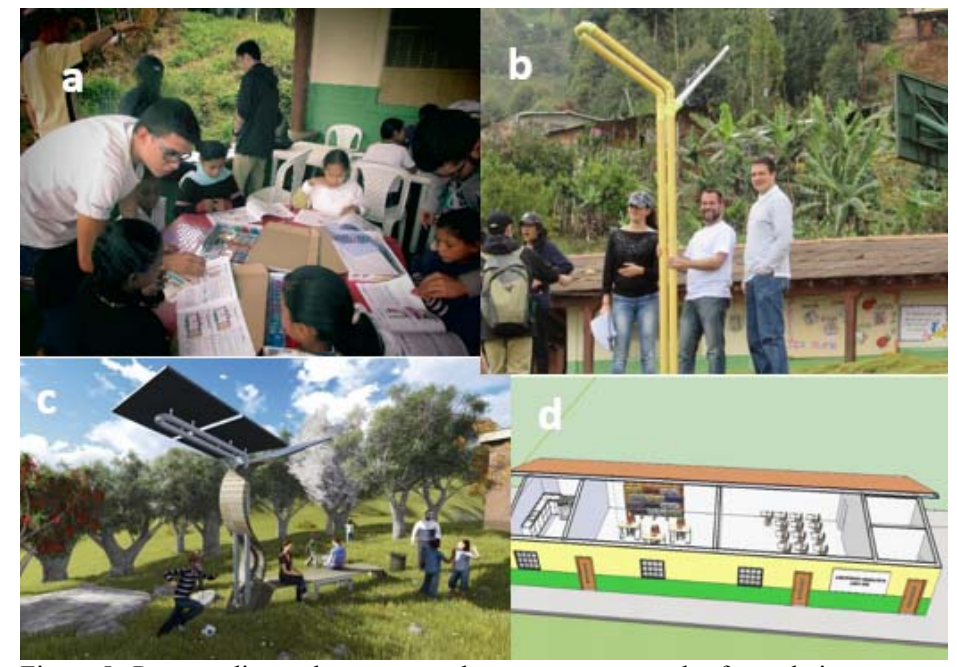

Figura 5. Proceso diseño de estructura de soporte para paneles fotovoltaicos: a. Encuentro con la comunidad y puesta en común del proyecto, b. Análisis de condiciones ambientales y socioculturales., c. Prototipo, socialización y ajustes., d. Diseño detallado final.

Fuente: Los autores.

\section{Resultados}

Como resultados directos de la aplicación de la metodología y la filosofía del curso PIIC, se han obtenido múltiples desarrollos de proyectos ingenieriles con impacto social, particularmente en las áreas del tratamiento de aguas y las energías renovables, sin embargo, la iniciativa PIIC ha participado en diversos proyectos de interés social, tal como se resume en la Fuente: Los autores.

En este sentido, es importante destacar que son varias las experiencias realizadas, los niveles de ejecución logrados y las empresas o grupos socios.

En la Figura 5 se ilustra a modo de ejemplo cómo son llevadas a cabo las distintas fases de un proyecto PIIC y en especial el de Energía Solar para Instituciones Educativas (IEs).

En el año 2016, los proyectos a realizar incluyen:

- Aulas virtuales: Aplicación de las nuevas tecnologías de realidad virtual a un entorno digital tridimensional para la transferencia de conocimiento.

- Filtros de arena a pequeña escala: Con la colaboración de Global Engineering [15] de la universidad de Purdue (EEUU), se han desarrollado propuestas de sistemas de filtrado de agua de bajo costo, a escala unifamiliar y para comunidades con deficiencias de acceso a agua potable. Estos filtros deben diseñarse a detalle, evaluarse a implementarse en la comunidad.

- Sistema de potabilización a mediana escala: Escalamiento de los sistemas desarrollados con Gobal Engineering para abastecer grupos de viviendas.

- Aulas biorreguladas: Diseño de hábitats térmicamente regulados con recubrimientos de plantas y tecnologías afines de bajo costo y ambientalmente amigables.

- Difusión de la vocación ingenieril: Diseño e implementación de estrategias para fomentar la vocación ingenieril en estudiantes de escuelas remotas o de bajos recursos. En este proyecto se cuenta con la colaboración de la universidad de Angelo State (EEUU), quienes visitarán Medellín en julio de 2017. 


\section{Conclusiones}

El esquema de trabajo logró generar un proceso en el que los proyectos que llegan a la fase de diseño de detalle, pasan a un banco de proyectos donde quedan a la espera de consecución de fondos o del perfeccionamiento de aspectos legales o administrativos y los alumnos, instituciones u organismos que participaron en su desarrollo recibirán siempre el reconocimiento, sin importar cuándo sea llevado a cabo la implementación final. Lo anterior, se convierte en una fuente de motivación adicional para estudiantes y grupos asociados que se sienten personalmente implicados en el buen término de sus proyectos.

El trabajo con instituciones internacionales conlleva sus propios retos, siendo los más complejos las diferencias idiomáticas y la coordinación de calendarios académicos no coincidentes. Estos retos obligan a estudiantes y docentes, a desarrollar habilidades comunicativas, más allá de lo usualmente requerido en sus actividades académicas cotidianas. Es usual que se forjen lazos interpersonales que sobreviven al término de los proyectos, con la consiguiente mejora en las posibilidades futuras de los estudiantes y la progresiva fluidez en las comunicaciones (y por lo tanto en la eficiencia de los grupos) que estas relaciones suponen.

Uno de los mayores desafíos de la ingeniería para el siglo XXI radica en abordar la reducción de la vocación ingenieril a nivel mundial, así como la alta tasa de deserción en carreras afines a la ingeniería y si bien los motivos de estas tendencias son dependientes de múltiples factores culturales, económicos y políticos, es innegable que uno de los determinantes principales es el nivel de vocación del estudiante, que a su vez define su nivel de compromiso y capacidad de afrontar los retos académicos y personales de su programa. Esta vocación no surge del vacío, sino que es el producto de la infiltración en el imaginario colectivo del papel de la ingeniería [16], no sólo como alternativa de movilidad social [17] (hay caminos al bienestar material percibidos como más sencillos), sino como una forma de participar de manera efectiva en la construcción del futuro equivalente y, en algunos casos, superior a otras más reconocidas. La iniciativa PIIC, junto con muchas otras que buscan el acercamiento entre el ingeniero y la sociedad, responde a esta necesidad de derribar barreras conceptuales y despejar prejuicios.

El éxito final en la lucha contra este declive vocacional dependerá de la suma de los esfuerzos de este tipo de iniciativas, por lo que es fundamental la creación de redes efectivas que integren y soporten a individuos y organizaciones a todos los niveles.

Un ejemplo inmediato del valor del apoyo institucional se vio claramente en la Facultad de Ingeniería de la Universidad de Antioquia en el año 2015, donde, tras la implementación de la UPI, se produjo un aumento considerable en la variedad de los proyectos y el número de estudiantes y alianzas estratégicas realizadas durante el curso PIIC. Se prevé que la tendencia continúe a medida que se implementan nuevas alianzas y campañas de difusión, lo que permitirá ejercer un impacto cada vez mayor, tanto en las comunidades como entre los estudiantes de la Universidad de Antioquia e Instituciones aliadas.
La realización de proyectos de ingeniería de carácter social, especialmente conducidos por estudiantes de pregrado y usando metodologías participativas, requiere el desarrollo y/o adopción de técnicas de evaluación de impactos y aprendizajes, no solo para los estudiantes, sino también para las comunidades. Este aspecto es fundamental para impulsar y justificar estas metodologías dentro de esquemas educativos tradicionales, donde se tiene a valorar prácticas evaluativas cuantitativas.

\section{Agradecimientos}

El desarrollo del curso PIIC será imposible sin el apoyo desinteresado de múltiples personas y grupos nacionales e internacionales. Entre ellos, y sin orden particular, los autores quisieran agradecer a:

- William Oakes, director del programa EPICS de la Universidad de Purdue.

- Chad Jafvert, profesor de la Universidad de Purdue y miembro de Global Engineering

- Ricardo Torres, director del Grupo de Investigación en Remediación Ambiental y Biocatálisis (GIRAB) de la Universidad de Antioquia

- Centro de Investigación, Innovación y Desarrollo de Materiales (CIDEMAT) de la Universidad de Antioquia

- Esperanza López Gómez, directora del Laboratorio de Mineralurgia Universidad de Antioquia

- Grupo Ingeniería y Sociedad de la Universidad de Antioquia.

- Grupo LUDENS de la Universidad de Antioquia.

- Alba Rúa, coordinadora Institución Educativa León XIII.

- Alejandro Roldán, profesional de cultura e innovación, Ruta N

\section{Referencias}

[1] ABET, Curricular Objectives, ABET 1993-94 Accreditation Yearbook, 1994.

[2] Garcia, F., Una mirada a la formación en ingeniería en el contexto internacional. Asociación Colombiana de Facultades de Ingenieria ACOFI, 2012.

[3] Cech, E.A., Culture of disengagement in engineering education?, Sci. Technol. Hum. Values. 39(1) pp. 42-72, 2013. DOI: 10.1177/0162243913504305

[4] Wall, K., Engineering: Issues, challenges and opportunities for development, UNESCO, 2010.

[5] Oakes, W. and Spencer, J., EPICS: Engineering projects in community service, in: Frontiers in Education, 2004. FIE 2004. 34th Annual, 2004 pp. 1455-1455. DOI: 10.1109/FIE.2004.1408794

[6] Facultad de Ingeniería, Transformación curricular documento rector. Universidad de Antioquia, 2006.

[7] Graaff E. D. and Kolmos, A., Characteristics of problem-based learning, Int. J. Eng. Educ., 19(5), pp. 657-662, 2003.

[8] Ander-Egg, E., Repensando la investigación-acción-participativa. Lumen-Humanitas, 2003.

[9] von Schomberg, R., A vision of responsible research and innovation, in: Responsible Innovation, R. Owen, J. Bessant, y Ggy Heintz, Eds. John Wiley \& Sons, Ltd, 2013, pp. 51-74. DOI:10.1002/9781118551424

[10] Pahl, G., Wallace, K. and Blessing, L. Engineering design: A systematic approach. Springer, 2007.

[11] Cross, N., Engineering design methods: Strategies for product design. Chichester, England; Hoboken, NJ: J. Wiley, 2008.

[12] Anderson D.J. y Reinertsen, D.G., Kanban: Successful evolutionary change for your technology business. Blue Hole Press, 2010. 
[13] Root, D., Trello Dojo. Leanpub, 2013.

[14] MINISTERIO DE EDUCACIÓN NACIONAL DIRECCIÓN DE POBLACIONES Y PROYECTOS INTERSECTORIALES. Lineamientos de política para la atención educativa a poblaciones vulnerables., jul-2005.

[15] Jafvert, C. and Howarter, J., Affordable slow sand filters. Personal communication, 2015.

[16] Changing the Conversation: Messages for Improving Public Understanding of Engineering. Washington, D.C.: National Academies Press, 2008

[17] EFA, Education for all: The quality imperative. UNESCO, 2015.
J.A. Vélez-Zea, recibe el titulo Ing. Mecánico en 2011 de la Universidad de Antioquia y el título de MSc. en Ingeniería Mecánica en 2014, de la Universidad Nacional de Colombia, Sede Medellín. Ha trabajado en programas y proyectos del área de ingeniería y sociedad con énfasis en enseñanza de la ingeniería. Se ha desempeñado como docente en la Universidad de Antioquia y actualmente es coordinador de la Unidad de Pedagogía en Ingeniería (UPI) de la misma universidad.

ORCID: 0000-0002-8467-359X

F. Jaramillo-Isaza, recibe el título de Ing. Químico en 2002, así como el Diplomado en Fundamentación Pedagógica y Didáctica Universitaria en el 2006, ambos de la Universidad de Antioquia. Adicionalmente es $\mathrm{PhD}$ en Química de la Universidad de Manchester, Inglaterra en 2005. Trabaja como profesor asociado del Departamento de Ingeniería de Materiales e Investigador activo del Centro de Investigación, Innovación y Desarrollo de MaterialesCIDEMAT, en la Sede de Investigación Universitaria-SIU de la Universidad de Antioquia. Miembro de la Red CYTED de Nanotecnologías para energía de la Región Iberoamericana - Red Nanoenergía. Fue consejero del Programa Nacional de Ciencia, Tecnología e Innovación de COLCIENCIAS 2013-2014. Representante de los coordinadores de grupos de investigación de máxima categoría ante el Centro de Investigación Ambiental - CIA, Facultad de Ingeniería, Universidad de Antioquia 2014-2015. Miembro de la iniciativa plataforma regional de nanotecnología nano $\mathrm{n}$, comisión académica para la generación de una política regional de nanotecnología y modelo de negocio; miembro de la Red nano Colombia y del Consejo Nacional Asesor de Nanociencia y Nanotecnología. Miembro del comité asesor para el Centro Nacional de Nanotecnología. Miembro del comité 243 del ICONTEC para la creación de Normas técnicas Colombianas en Nanotecnología. ORCID: 0000-0003-1722-5487

J.C. Saldarriaga-Molina, recibe el título de Ing. Sanitario en 1995 . Adicionalmente sus títulos de MSc. en Ingeniería Ambiental y Dr. en Ingeniería de la Universidad de Antioquia en 2001 y 2011 respectivamente. Ha trabajado como profesor de tiempo completo adscrito a la Escuela Ambiental de la Facultad de Ingeniería y ha ocupado algunos cargos administrativos como: Jefe del Departamento de Ingeniería Sanitaria y Ambiental; Coordinador de Posgrados y Vicedecano de la misma Facultad en los últimos cinco años y medio. Actualmente, ocupa el cargo de coordinador de la Unidad de Virtualidad de la UdeA.

ORCID: 0000-0002-9395-5417

C.A. Palacio-Tobón, recibe el título de Ing. Civil en 1994 de la Universidad Nacional de Colombia, Sede Medellín, el título de MSc. en Ingeniería Civil en 1996 de la Universidad de Los Andes, Bogotá, y el título de Dr. en Ingeniería, Área Aprovechamiento de Recursos Hidráulicos, de la Universidad Nacional de Colombia, Sede Medellín, en programa conjunto con la Universidad Christan Albrecht de Kiel, Alemania, en el 2002. Desde el año 2002 hasta la fecha ha estado vinculado como profesor de la Universidad de Antioquia, donde ha asumido el cargo de vicedecano de la Facultad de Ingeniería entre 20072010, Decano de Facultad de Ingeniería entre 2010-2016, y actualmente en el cargo de Vicerrector de Extensión de la Universidad de Antioquia. Ha sido docente de pregrado y posgrado e investigador en el área de mecánica de fluidos, métodos numéricos, física atmosférica, oceanografía física, modelación atmosférica, modelación Oceanográfica y modelación de calidad del agua. ORCID: 0000-0001-7932-7046 